\title{
Solving the Organ Shortage Crisis: The 7th Annual American Society of Transplant Surgeons' State-of-the-Art Winter Symposium
}

\author{
E. A. Pomfret ${ }^{a, *}$, R. S. Sung ${ }^{b}$, J. Allan ${ }^{c}$, M. \\ Kinkhabwala $^{d}$, J. K. Melancon ${ }^{\mathrm{e}}$ and J. P. Roberts ${ }^{\mathfrak{f}}$
}

a Division of Liver Transplantation and Hepatobiliary Surgery, Lahey Clinic Medical Center, Burlington, MA ${ }^{\mathrm{b}}$ Division of Transplant Surgery, University of Michigan Health Center, Ann Arbor, MI

${ }^{c}$ Department of Surgery, Division of Thoracic Surgery, Massachusetts General Hospital, Boston, MA

'Division of Transplantation, Montefiore Medical Center, Bronx, NY

e Division of Transplant Surgery, Johns Hopkins Medical Institute, Baltimore, MD

${ }^{f}$ Department of Surgery, Division of Transplantation, University of California, San Francisco, San Franciso, CA

* Corresponding author: Elizabeth A. Pomfret,

Elizabeth.A.Pomfret@Lahey.org

The 2007 American Society of Transplant Surgeons' (ASTS) State-of-the-Art Winter Symposium entitled, 'Solving the Organ Shortage Crisis' explored ways to increase the supply of donor organs to meet the challenge of increasing waiting lists and deaths while awaiting transplantation. While the increasing use of organs previously considered marginal, such as those from expanded criteria donors (ECD) or donors after cardiac death (DCD) has increased the number of transplants from deceased donors, these transplants are often associated with inferior outcomes and higher costs. The need remains for innovative ways to increase both deceased and living donor transplants. In addition to increasing ECD and DCD utilization, increasing use of deceased donors with certain types of infections such as Hepatitis B and C, and increasing use of living donor liver, lung and intestinal transplants may also augment the organ supply. The extent by which donors may be offered incentives for donation, and the practical, ethical and legal implications of compensating organ donors were also debated. The expanded use of nonstandard organs raises potential ethical considerations about appropriate recipient selection, informed consent and concerns that the current regulatory environment discourages and penalizes these efforts.

Key words: Access to transplantation, deceased donor kidneys, deceased donor organs, donor/recipient matching, ethics, expanded criteria donors, live donor transplantation, organ donation, organ sales, organ shortage

Received 05 September 2007, revised 07 December 2007 and accepted for publication 17 December 2007

\section{Introduction}

Organ transplantation has in some ways become a victim of its success. Life-saving organ replacement therapy has been offered to an increasingly complex population of patients with an increasing success rate. As a result, the number of transplants performed annually is dwarfed by the number awaiting transplantation. In addition, the number of deaths that occur while awaiting transplantation approaches the number of patients transplanted each year (1). Currently, there is an ongoing increase in the number of patients who are awaiting kidney transplantation, while the number of patients on the liver transplant list has been stable. Despite this stability, there are ongoing deaths among patients on the waiting list. The transplantation community has responded by developing various strategies to expand the pool of available organs including the utilization of extended criteria donors (ECD), donation after cardiac death (DCD) and live donor (LD) transplantation. The 7th Annual American Society of Transplant Surgeons State-of-the-Art Winter Symposium explored the implications of expanding the donor pool in this manner including donor and recipient risk factors, the concept of 'net benefit', outcomes and ethical considerations associated with implementation of these strategies.

The scope of organ donation and the complexity of potential solutions to the shortage of organs for transplantation preclude comprehensive reporting of all possible solutions in this report. We have attempted to present a balanced representation of the information discussed at the symposium. However, many of the strategies reviewed are proposals or in the very early stages of clinical implementation. Some of the information represents the opinions of the individual speakers, and while these opinions are well informed, we acknowledge that some of the opinions are likely to be subject to debate.

\section{Defining the problem}

Although the number of donors in the United States has increased steadily over the past decade, the age and source 


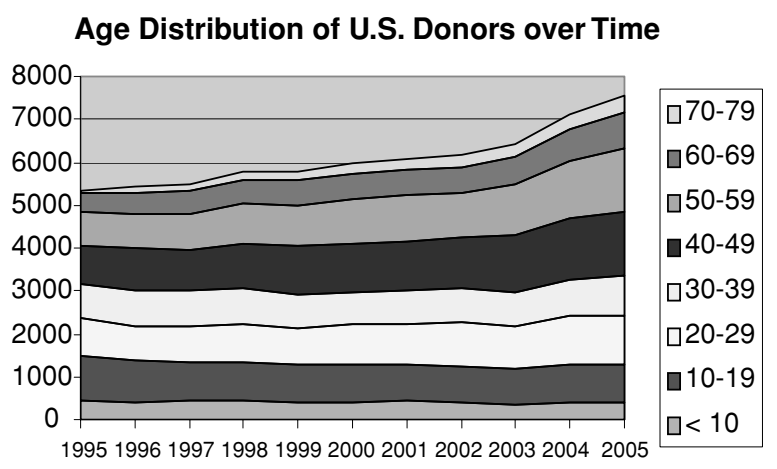

Figure 1: Age distribution of US donors over time. The change in age distribution of organ donors that has occurred in the United States over the past decade is shown. Donors under the age 40 years have remained stable while donors over 40 years have increased significantly.

distribution have undergone significant changes. The effect of these changes was reviewed by John Roberts (University of California, San Francisco). Currently, more than $60 \%$ of donors are 40 years of age or older while the number of younger donors has remained relatively static (Figure 1) (1). This finding is similar to that previously observed in Spain when a marked increase in the number of donors occurred during the 1990s (2). Examination of the US data also demonstrates that a significant portion of the increased donation from younger donors is the result of increased utilization of DCD donors (3). While the use of DCD and older donors has obviously increased the number of organs available for transplantation, the practice presents new challenges to both the recipients of the expanded criteria organs and their physicians.

Robert Merion of the University of Michigan discussed the evolution of death on the waiting list. To some extent the use of marginal organs has been successful in preventing waitlist deaths, since their number has remained stable since 2002. While the number of deaths of candidates awaiting kidney transplant has continued to increase, the numbers have decreased for heart, lung, and heart-lung transplant candidates. The number of deaths among candi-

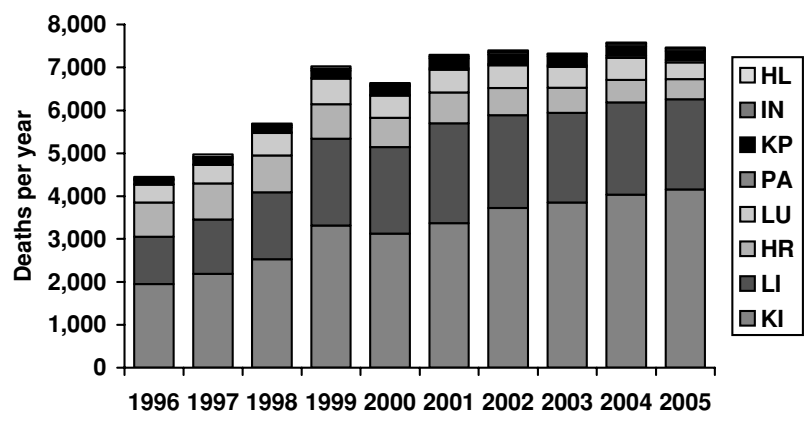

Figure 2: Waiting list deaths by organ, 1996-2005. The incidence of death while awaiting transplant for various categories of candidates is shown. Deaths among kidney candidates have steadily increased, while a decrease was seen for heart, lung or heart lung candidates. The death rates while waiting have been relatively stable for other types of candidates.

dates awaiting liver, pancreas, combined kidney-pancreas or intestinal transplantation has remained relatively stable (Figure 2, Table 1). Despite these changes in the number of waiting list deaths by organ, death rates (i.e. numbers of deaths per patient-years at risk) for heart, liver and kidney candidates have remained the same since 2000.

The assessment of waiting list deaths is complicated by the significant and increasing percentage of inactive waiting list candidates. These candidates, who comprised over $20 \%$ of the kidney and liver waiting lists in 2005, have higher mortality risk than those candidates who are active (adjusted HR = 2.31 for kidney, 4.87 for liver, SRTR Special Analysis, 2007). However, because a significant majority of listed patients are active, deaths among inactive candidates contribute less to the overall death rates on the waiting list than do those of active candidates (Figures $3 \mathrm{~A}$ and $\mathrm{B}$. In addition, death rates for inactive liver and kidney candidates are decreasing; this (especially in light of the increase in the percentage of inactive candidates) may be a consequence of changes in waiting list status management rather than improvements in pretransplant care. These trends suggests that a significant proportion of waitlist deaths continue to be among those candidates who

Table 1: Number of candidate deaths on waitlist by organ per year

\begin{tabular}{|c|c|c|c|c|c|c|c|c|c|c|}
\hline & 1996 & 1997 & 1998 & 1999 & 2000 & 2001 & 2002 & 2003 & 2004 & 2005 \\
\hline \multicolumn{11}{|l|}{ Organ } \\
\hline Kidney & 1949 & 2184 & 2528 & 3320 & 3125 & 3375 & 3729 & 3853 & 4038 & 4156 \\
\hline Liver & 1103 & 1274 & 1565 & 2017 & 2018 & 2324 & 2160 & 2087 & 2145 & 2104 \\
\hline Heart & 802 & 838 & 859 & 804 & 686 & 716 & 631 & 586 & 525 & 465 \\
\hline Lung & 420 & 437 & 520 & 600 & 518 & 532 & 529 & 489 & 514 & 394 \\
\hline Pancreas & 7 & 11 & 21 & 14 & 17 & 35 & 30 & 35 & 51 & 55 \\
\hline Kidney/Pancreas & 95 & 124 & 108 & 169 & 198 & 228 & 223 & 208 & 224 & 206 \\
\hline Intestine & 24 & 44 & 48 & 47 & 27 & 44 & 52 & 48 & 56 & 57 \\
\hline Heart-Lung & 52 & 63 & 43 & 55 & 46 & 42 & 42 & 20 & 29 & 27 \\
\hline Total & 4452 & 4975 & 5692 & 7026 & 6635 & 7296 & 7396 & 7326 & 7582 & 7464 \\
\hline
\end{tabular}


A

Figure 3: (A) Kidney waiting list death rates by active inactive and year, 1996-2005, and (B) Liver waiting list deaths by active inactive and year, 1996-2006. The effect of death among inactive candidates on overall waitlist mortality for kidney (A) and liver (B) candidates is demonstrated. Although the death rates among active candidates are significantly greater for both groups ( $p=$ $<0.05$ ) compared to deaths in the total or the active cohorts, the number of inactive candidates is small and removal does not result in significant differences in the death rates between the total and active candidates.

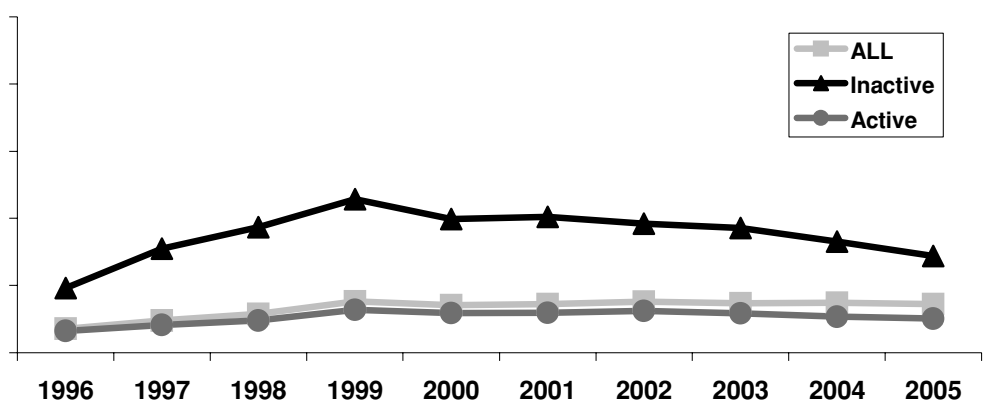

B

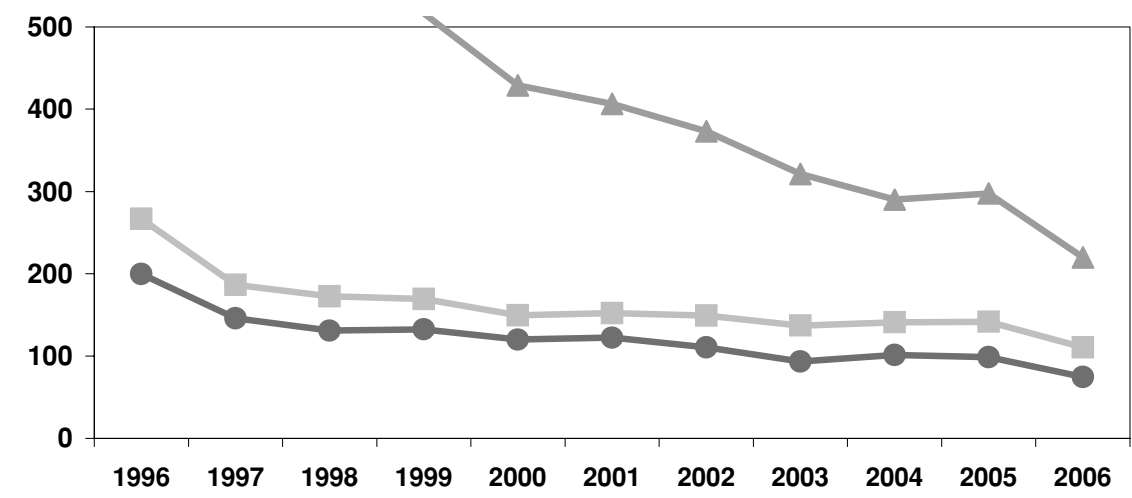

were intended to, and were in fact eligible to, receive a transplant.

One challenge is that transplantation with ECD or DCD organs results in significant cost increases compared to transplantation with standard criteria donors. Data presented by Schnitzler (St. Louis University) demonstrated that kidney transplantation results in a cost saving when compared to dialysis, and that live donor kidney transplantation with its shorter waiting times, less dialysis time and improved organ quality enhances the cost savings (Figure 4) (4). However, when cost savings is examined in terms of donor organ source, differences are apparent (Figure 5). While transplantation is less costly than dialysis in all instances, transplantation with standard criteria kidneys results in significantly greater savings as measured by dollars per quality-adjusted life-year than transplantation with either DCD or ECD kidneys (Figure 3). A similar effect is seen in liver transplantation; patients transplanted with DCD livers had significantly increased length of stay and therefore costs when compared to those receiving standard criteria livers (Table 2).

A second challenge that arises with the utilization of nonstandard donor organs is: 'Which patients should be offered these organs?' There is agreement that patients who would benefit from transplantation can be accurately identified. There is, however, no consensus regarding the type and quality of organs that should be offered to various recipients. Several of the participants reviewed exist- ing practices and strategies designed to answer these questions.

John Magee (University of Michigan) outlined the ethical considerations involved in various organ allocation schemes and reviewed the existing data. He pointed out that any allocation scheme would be complicated by the fact that the transplant community's perception of benefit and a transplant patient's perception of benefit is likely to differ in many instances. As an example he offered the case of a patient given a $5 \%$ chance of survival without transplantation and a $20 \%$ chance of survival with transplantation. The patient will see transplantation, as being in his best interest while the transplant provider will likely have greater doubts. Some allocation proposals focus on the benefit potential of a donor organ as a significant factor in recipient selection. These proposals would allocate an organ with a potential survival of 30 years to recipients with similar expected longevity. The ideal allocation scheme would balance individual justice, instances of urgency, and utility-doing the most good for most of the people. While the ideal was agreed upon, the mechanics of achieving it remained elusive despite considerable discussion.

\section{Outcomes in recipients of nonstandard donors}

For adult liver transplantation, Feng et al. (5) have developed the 'donor risk index' (DRI)—a semi-quantitative estimate of the likelihood of liver failure following 


\section{Cost Saving From Kidney TX}

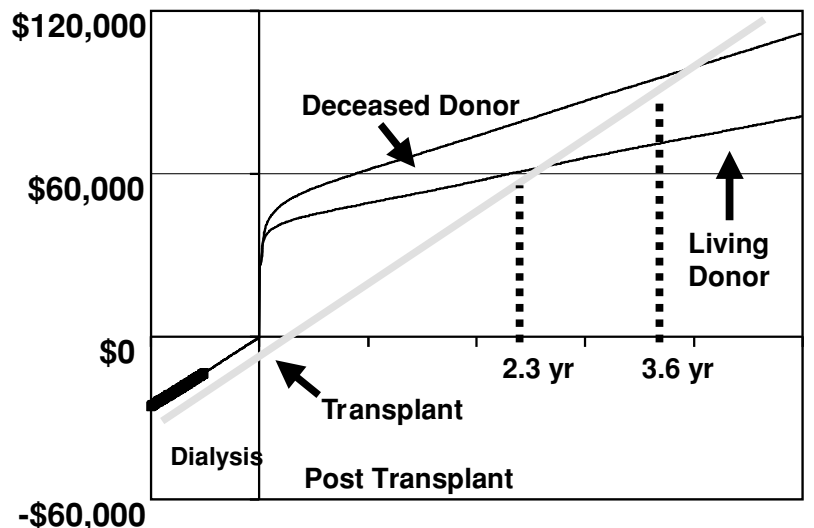

Transplantation 67(7):S189. 1999

Figure 4: Cost savings of various types of kidney transplant compared to maintenance dialysis. Cost savings achieved by transplantation with either a deceased or living donor kidney compared to maintenance dialysis.

transplantation. They showed that factors such as donor age, DCD status and use of partial liver grafts are associated with a greater risk of graft loss than transplantation with a standard criteria donor (SCD) liver. Risks are categorized as high, medium or low. Two distinct schools of thought have emerged regarding the use of higher risk organs. The first is to transplant these livers into patients with low MELD scores in the hope that their relative good health will allow them to withstand poor allograft function and still survive. The alternative approach is to use these organs in sicker patients with higher MELD scores because they are more likely to die without a liver transplant.

The concern voiced about using the higher risk livers in sicker patients is that they would do less well with these grafts because they would lack the physiologic reserves to withstand poor function. Data from Schaubel et al. (6) examined the effect of DRI scores on survival across all MELD ranges. Figure 4 compares the effect of remaining on the waitlist to transplantation with a low, medium or high DRI liver at various MELD scores. He showed that at any given MELD score, use of a high DRI liver pro-

Table 2: Impact of DCD donor on liver transplantation costs

\begin{tabular}{llll}
\hline $\begin{array}{l}\text { MELD } \\
\text { category }\end{array}$ & \multicolumn{2}{l}{ Length of stay (Days) } & \multicolumn{1}{c}{$\begin{array}{c}\text { Estimated } \\
\text { cost increase }\end{array}$} \\
\cline { 2 - 4 } & DCD & DBD & $\$ 2264$ \\
\hline $0-10$ & 18.1 & 17.6 & $\$ 12681$ \\
$11-20$ & 19.0 & 16.2 & $\$ 9058$ \\
$21-30$ & 20.8 & 18.8 & $\$ 26268$ \\
$31-35$ & 34.7 & 28.9 & $\$ 8152$ \\
$>35$ & 42.7 & 40.9 & \\
\hline
\end{tabular}

\$ / QALY

Simultaneous ECD, DCD \& Std. TX Compared to Life on Dialysis

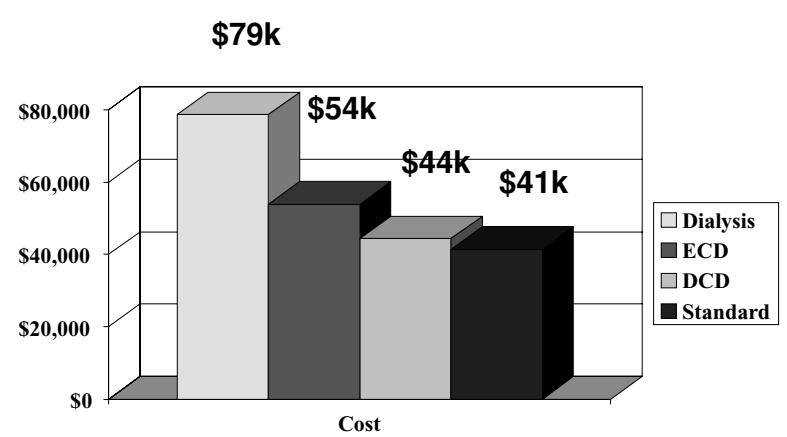

Figure 5: Costs associated with transplantation of standard criteria versus ECD or DCD kidneys. Comparison of costs associated with transplantation with standard, ECD or DCD kidneys compared to ongoing dialysis. All types of transplantation result in cost savings compared to dialysis $(p<0.05)$, but transplantation with standard criteria kidney results in significant $(p<0.05)$ cost savings compared to either DCD or ECD transplantation.

duced a worse outcome compared to a low DRI organ. At low MELD scores, there is no survival benefit obtained from liver transplantation and this is exacerbated when high DRI livers are used. However, in patients with high MELD scores, transplantation with livers from any DRI category provided significant survival advantage compared to remaining on the waitlist.

Randall Sung (University of Michigan) reviewed the results of the recently implemented ECD allocation system for kidney transplantation. Recovery and transplantation of ECD kidneys have increased since implementation, while delayed graft function rates and graft survival have not changed (7). There is no association between transplant outcome and either biopsy findings or machine perfusion, but these two factors continue to be important determinants of ECD kidney discard (8). There is also great variation in discard rates of ECD kidneys by geographic areas (Figure 6). This suggests that maximal utilization of ECD kidneys requires development of mechanisms to shift these organs quickly from low to high utilization areas. While there is a disadvantage for graft survival that applies equally to all categories of ECD kidney recipients compared to SCD organs, benefit analyses suggest a survival advantage to certain subgroups. Specifically, older and/or diabetic recipients in regions with prolonged waiting times demonstrate a significant survival increase compared to waiting for a SCD kidney (9). The proposed kidney allocation scheme based on the concept of 'net benefit' does not include ECD kidneys. Therefore, ECD kidneys may represent an important alternative for those patients less likely to receive a kidney transplant under a revised SCD algorithm. A need for the development of a continuous kidney donor risk index was identified so that more precise risk information could be presented at the time of organ offer. 


\section{Transplant Survival Benefit By MELD and Donor Risk Index}

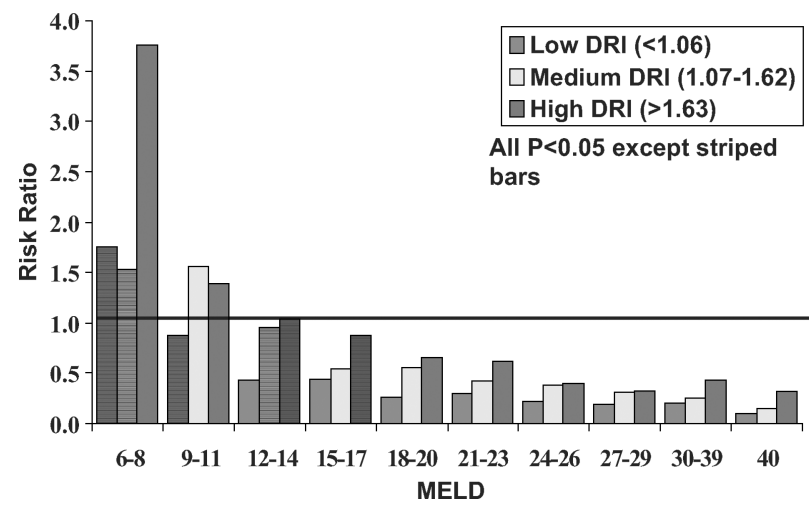

Figure 6: Transplant survival benifit by MELD and donor risk index. Comparison of the survival benefit of liver transplantation by MELD and DRI. At the same MELD scores, high DRI organs produced worse outcomes than low DRI organs. At low MELD scores, high DRI organs produce no survival benefit. However, at high MELD scores, transplantation with any DRI offers survival benefit compared to continued waiting on the list.

Jeevanandam (University of Chicago) discussed the use of ECD donors in heart transplantation. As was seen in kidneys and livers, the use of an ECD heart was associated with inferior results compared to SCD hearts. However, survival following ECD heart transplantation is clearly superior to remaining on the waiting list or treatment with a left ventricular assist device. Spurred on by the significant short term waiting list mortality, heart transplant centers at the University of Chicago and UCLA, have begun to use hearts from donors over 50 , those with CAD, poor function or inotrope use, size mismatches and long preservations times. This has resulted in a $33 \%$ increase in the number of donors. The creation of alternative lists of patients willing to take these organs has ensured efficient use of the available organs. Additional important principles include avoidance of multiple recipient risk factors, optimization of cardiac performance prior to recovery and revascularization of identified coronary lesions prior to transplant (10-12).

The complexity of evaluating potentially infected donors was discussed by Emily Blumberg (University of Pennsylvania). Bacterial infections are not considered to be a contraindication for donation unless the donor organ is affected and published guidelines for the treatment of potentially infectious donors are available (13). The proper course is less clear when the known or suspected infectious agent is viral. Some, such as hepatitis B or C, are organ specific and may be suitable for use in some patients. The impact of less common infections, such as HTLV 1 and 2 is unknown. The experience with West Nile Virus (WNV) illustrates the dilemma of screening for uncommon or emerging infections in potential organ donors. The virus may cause meningoencephalitis, but is usually asymptomatic in normal individuals. However, in recipients of organs from WNV positive donors, a 40-fold increase in the incidence of neuroinvasive disease is noted and outcomes are dismal. While nucleic acid testing for WNV exists, its availability and reliability are variable, costly and identify few cases. Exclusion of donors from endemic areas would greatly reduce an already insufficient organ supply and is not advisable given the very low rate of actual infections. Current recommendations are to avoid organ donation from patients with undiagnosed febrile illnesses, encephalitis, meningitis or flaccid paralysis of unknown etiology. Future efforts should focus on development of rapid and accurate diagnostic tests.

The special circumstances encountered when considering use of a live donor liver graft from an individual infected with hepatitis B were reviewed by Chung Mao Lo (Queen Mary Hospital, Hong Kong). In this instance, donor safety must be considered as well as recipient outcome. In deceased donor liver transplantation, anti-HBc(core) positive, $\mathrm{HBs}$ (surface) antigen negative donor organs are routinely offered to HbsAg positive recipients. Reactivation of hepatitis $B$ virus is expected in immunosuppressed individuals, and does not appear to differ regardless of donor source. However, in LDLT, both graft and remnant need to regenerate, and the effect of hepatic regeneration on viral reactivation is not well studied. Their experience suggests that liver function and hepatic regeneration in both the donor and recipient is not affected by donor seropositivity for HBc (14-16). This example also highlights the need to factor cultural realities into allocation schemes. In Asia, where deceased donor transplantation is a limited option and the prevalence of hepatitis B exposure is large, exclusion of anti-HBc positive donors would significantly reduce the rates of transplantation.

\section{Living donors}

The shortage of deceased donor organs has led to the predictable expansion of live donor transplantation for most categories of recipients. Kidney transplantation has the greatest experience in the use of living donors-the number of live donor kidney transplants frequently exceeds the number of deceased donor transplants in a reporting period (1). Despite this and the increased use of ECD donors described above, patients continue to be waitlisted for extended periods. Dorry Segev (Johns Hopkins) described strategies that help patients with willing, but incompatible, living donors. Plasmapheresis/intravenous immunoglobulin/anti-CD20 can be used to eliminate or reduce to manageable levels the antibodies preventing transplantation in $\mathrm{ABO}$ or HLA crossmatch incompatible pairs (17-19). Another potential means for increasing transplant rates is 'paired donation' in which two or more donorrecipient pairs with reciprocal incompatibilities, agree to exchange donated organs. This results in a net gain of 2 donor organs and removal of 2 recipients from the waiting 
list $(20,21)$. A variety of matching algorithms and consortia have developed, which increases the likelihood of identifying a match for individual donor-recipient pairs, and also increases the possibility of having exchanges among multiple pairs, or 'domino' paired donation (22).

The use of living donors in liver transplantation is increasing, especially in areas with long waitlist times (2). The overall incidence of live donor liver transplants (LDLT) peaked at $10 \%$ of the total number of liver transplants performed in 2001 (3). Since then, the percentages have decreased to $5 \%$ of the total ( 320 LDLT/year). In regions where the average MELD score for DDLT is 25 or greater (Regions 1, 5, 7 and 9), the percentage of patients undergoing LDLT continues to increase and currently represents approximately $10 \%$ of all liver transplants. Kim Olthoff (University of Pennsylvania) reviewed the results obtained thus far in the Adult to Adult Living Liver (A2ALL) donor transplantation study (23-28). Older recipient age and center experience of less than 20 cases was associated with increased graft failure. LDLT is associated with reduced waitlist mortality and posttransplant patient and graft survival is equivalent to deceased donor liver transplantation (DDLT) in experienced centers. Rejection rates, HCV recurrence and cumulative risk of fibrosis were also similar. Recurrence free survival for patients transplanted for hepatocellular carcinoma was significantly lower for LDLT recipients compared to DDLT. This may reflect the larger number of patients with T3 pathology on their explants.

Olthoff also reported on donor morbidities experienced after donor hepatectomy. Overall rehospitalization rate was $12 \%$ while the overall complication rate was $38 \%$. Specific complications included bile leaks (9.2\%), hernias (5.6\%), psychological (4\%) and pulmonary (6\%). The majority of donor complications was Clavien grade 1 or 2 with only $2 \%$ experiencing grade 3 complications. There were 4 donor deaths during the study period but only one was donation related. The others were late occurring (accident, drug over dose and suicide) (29).

Utilization of living donor intestinal transplantation was reviewed by Benedetti (University of Illinois, Chicago). He cited that mortality on the waitlist was higher than for any other organ, especially in the pediatric age group. Advantages of live donation including reduction of ischemicreperfusion injury, potential for better HLA matching and optimal gut/graft decontamination were highlighted. Disadvantages included potential harm to the donor, technically difficult implantation due to a shorter vascular pedicle and a smaller graft. Combined pediatric liver-intestine grafts, either simultaneous or sequential, had better outcomes than recipients of isolated small bowel grafts. Recipients of combined grafts $(n=5)$ reported only 1 graft failure, and no rejection episodes or patient deaths while 2 recipients of isolated small bowel grafts $(n=5)$ died from subsequent liver failure.
Mark Barr (University of Southern California) discussed the use of living donors in lung transplantation $(30,31)$. This procedure is unique in that it requires two donors for each recipient; one donor will give a right lower lobe while the other donates a left lower lobe. The most common recipient diagnosis is cystic fibrosis (86\%). Comparable survival rates are observed in adult and pediatric recipients and no significant difference from recipients of deceased donor grafts is observed. Recipients of live donor lobar transplants appear to have a decreased incidence of bronchiolitis obliterans at all time points compared to published incidence in deceased donor recipients. Ventilator dependence at time of transplant was associated with poor outcome. He reports that $80 \%$ of donors had no perioperative complications. Donor complications occurred more often in right lobe donors, and the most common complication was need for a thoracostomy tube for greater than 14 days. Long-term follow-up of donor outcomes and functional effects is recommended.

\section{Ethical issues arising from the organ shortage crisis}

Any allocation strategy for a life-saving scarce resource will be unavoidably entwined with ethical issues given that the end result of the strategy is that while some lives will be saved, others will not. This reality was reflected in the content of many presentations and the discussions that followed. James Childress (University of Virginia) presented the Institute of Medicine (IOM) report on increasing the rates of organ donation (32). They concluded that presumed consent for organ donation is not currently a realistic approach in the United States and that emphasis should be on including organ donation as part of quality end-of-life care plans. He reported that the IOM did not recommend implementation of incentivised donation at this time, although he did not rule out future implementation. The report also recommended exploration of ways to utilize people who succumb to out-of-hospital cardiac arrest as donors.

The need to provide patients informed consent regarding the quality of the organ being offered for transplant was discussed in several talks. Drs. Arthur Caplan and James Allan had a lively debate on this subject with Dr. Caplan arguing for complete disclosure. He felt that the principles of patient autonomy and dignity require nothing less, especially when potentially risky therapies are offered; to do less undermines the trust that is the cornerstone of the doctor-patient relationship (33). Dr. Allan acknowledged a patient's right to autonomy, but argued that society routinely limits individual autonomy when it interferes with the greater good. He cited triage of medical services at disaster scenes noting that some are forced to wait while the available medical care is directed toward others in more urgent need (34). Kinkhabwala (Montefiore) suggested disclosing what a reasonable person would need to know to make an informed decision, including the success rates 
with the type of organ in question. He suggested that the discussion occur during the evaluation/listing process and that consenting patients be placed on both standard and alternative lists (35). John Renz (NY-Presbyterian) pointed out that transplantation with high-risk organs such as those infected with HTLV might require formal IRB approval in addition to patient consent (35).

Finally, the ethics of incentivised donation were debated by Arthur Matas (University of Minnesota) and Frank Delmonico (Massachusetts General Hospital). Matas suggested a regulated system of organ 'sales' that would insure adequate safeguards to both the donor and the quality of organ procured. He argued that the result would be an increase in the overall number of transplants and a corresponding decrease in the number of transplant preventable deaths (36). Delmonico argued against the practice, citing the increase in donations in recent years, the potential for additional increases with increased donor awareness education and the significant decreases in familial donation documented in systems allowing overt organ sales (37, 38).

\section{Summary}

The symposium provided transplant professionals an opportunity to explore various options for dealing with the inadequate supply of organs for transplantation. It is clear that living donation and non-standard deceased donation are the solutions in current favor. Once the transplant team has sufficient experience, the results for recipients of living donor organs are equal or superior to those for standard criteria deceased donors. The short-term results for recipients of ECD donor organs are inferior to those obtained with SCD donors, but appear acceptable in many instances considering the high mortality associated with remaining on the waitlist. However, the true value of this strategy cannot be assessed until long-term follow-up is available.

\section{References}

1. UNOS data. Available at: http://www.unos.org. Accessed January 2007.

2. Chang GJ, Mahanty HD, Ascher NL, Roberts JP. Expanding the donor pool: Can the Spanish model work in the United States? Am J Transplant 2003; 3: 1259-1263.

3. Pomfret EA, Fryer JP, Sima CS, Lake JR, Merion RM. Liver and intestine transplantation in the United States, 1996-2005. Am J Transplant 2007; 7(Suppl 1): 1-14.

4. Schnitzler MA, Smith C, Woodward RS et al. Relative cost of cadaveric versus living donor kidney transplantation (Abstract 729). Transplantation 1999; 67: S189.

5. Feng S, Goodrich NP, Bragg-Gresham JL et al. Characteristics associated with liver graft failure: The concept of a Donor Risk Index. Am J Transplant 2006; 6: 783-790.

6. Schaubel DE, Sima CS, Goodrich NP et al. Survival benefit of transplantation by MELD and Donor Risk Index. Am J Transplant 2006; 6(Suppl 2): 95.
7. Sung RS, Guidinger ML, Lake $C$ et al. Impact of the expanded criteria donor (ECD) kidney allocation system on the use of ECD kidneys. Transplantation 2005; 79: 1257-1261.

8. Sung RS, Guidinger MK, Christensen LL et al. Development and current status of ECD kidney transplantation. In: Cecka JM, Terasaki PI (eds). Clinical Transplants. Los Angeles: UCLA Tissue Typing Laboratory, 2006: 37-55.

9. Merion RM, Ashby VB, Wolfe RA et al. Deceased donor characteristics and the survival benefit of kidney transplantation. JAMA 2005; 294: 2669-2673.

10. Marelli $D$, Laks $H$, Bresson J et al. Sixteen year experience with 1,000 heart transplants at UCLA. In: Cecka JM, Terasaki PI (eds). Clinical Transplants. Los Angeles: UCLA Tissue Typing Laboratory, 2000: 297-310.

11. Jeevanandam V, Furukawa S, Prendergast TW et al. Standard criteria for an acceptable donor heart are restricting heart transplantation. Ann Thor Surg 1996; 62: 1268-1275.

12. Young JB, Naftel DC, Bourge RC et al. Matching the heart donor and the heart recipient. Clues for successful expansion of the donor pool: A multivariate multiinstitutional report. The Cardiac Transplant Research Database Group. J Heart Lung Transplant 1996; 10: 1468-1475.

13. Angelis M, Cooper JT, Freeman RB. Impact of donor infections on the outcome of orthotopic liver transplantation. Liver Transplant 2003; 9: 451-462.

14. Kiuchi T, Kashahara M, Uryuhara K et al. Impact of size mismatching on graft prognosis in liver transplantation from living donors. Transplantation 1999; 67: 321-327.

15. Lo CM, Fan ST, Liu CL et al. Safety and outcome of hepatitis B core antibody positive donors in right-lobe living donor liver transplantation. Liver Transplant 2003; 9: 827-832.

16. Hwang S, Moon DB, Lee SG et al. Safety of anti-hepatitis B core antibody positive donors for living donor liver transplantation. Transplantation 2003; 75: S45-S48.

17. Segev DL, Simpkins CE, Warren DS et al. ABO incompatible hightiter renal transplantation without splenectomy of anti-CD20 treatment. Am J Transplant 2005; 5: 2570-2575.

18. Jordan S, Cunningham-Rundles C, McEwan R. Utility of intravenous immune globulin in kidney transplantation: efficacy, safety, and cost implications. Am J Transplant 2003; 3: 653-664.

19. Stegall MD, Gloor J, Winters JL, Moore SB, Degoey S. A comparison of plasmapheresis versus high-dose IVIG desensitization in renal allograft recipients with high levels of donor specific alloantibody. Am J Transplant 2006; 6: 346-351.

20. Segev DL, Gentry SE, Warren DS et al. Kidney paired donation and optimizing the use of live kidney donors. JAMA 2005; 293: 1883-1990.

21. Gentry SE, Segev DL, Montgomery RA. Comparison of populations served by kidney paired donation and list paired donation. Am J Transplant 2005; 5: 1914-1921.

22. Montgomery RA, Gentry SE, Marks WH et al. Domino paired kidney donation: A strategy to make best use of live non-directed donation. Lancet 2006; 368: 419-421.

23. Olthoff KM, Abecassis MM, Ghobrial RM et al. Factors associated with graft failure and outcome in 354 adult living liver donor transplants (ALDLT): A report from the A2ALL consortium. Ann Surg 2005; 242: 314-323.

24. Berg $C L$, Abecassis MM, Brown RS et al. Living donor liver transplantations reduces the risk of death of transplant candidates. Hepatology 2004; 40(Suppl 1): 209A.

25. Berg CL, Gillespie BW, Merion RM et al. Adult living donor liver transplantation reduced transplant candidate mortality. Am J Transplant 2006; 6(Suppl 2): 172. 


\section{Pomfret et al.}

26. Kulik LM, Freise CE, Lok AS et al. A comparison of adult living donor (LDLT) to deceased donor liver transplant (DDLT) for hepatocellular carcinoma (HCC): Data from the A2ALL study. Hepatology 2005; 42(Suppl1): 53A.

27. Terrault NA, Shiffman ML, Lok AS et al. Transplant center experience explains differences in the risk of graft failure between hepatitis $C$ infected living donor (LDLT) and deceased donor (DDLT) liver transplant recipients. Hepatology 2005; 42(Suppl 1): 53A.

28. Shaked A, Hulbert-Shearon TE, Merion RM et al. Biopsy-proven rejection rate is equivalent in recipients undergoing living versus deceased donor liver transplantation. Am J Transplant 2005; 6(Suppl 6): 172.

29. Ghobrial RM, Freise CE, Trotter JF et al. Donor morbidity and mortality of adult living donors for liver transplantation. Am J Transplant 2005; 6(Suppl 2):115.

30. Bowdish ME, Barr ML. Living lobar transplantation. Respir Care Clin N Am 2004; 10: 563-570.

31. Bowdish ME, Barr ML, Schenkel FA et al. A decade of living lo- bar transplantation: Perioperative complications after 253 donor lobectomies. Am J Transplant 2004; 4: 1283-1290.

32. IOM Report on Increasing Rates of Organ Donation: Opportunities for Action. Washington, DC: National Academies Press, 2006.

33. Reese PR, Bloom RD, Caplan AL. Creating a medical, legal and ethical framework for complex kidney donors. JASN 2006; 11: 11481153.

34. Veatch RM. A General Theory of Organ Allocation. Washington, DC: Georgetown University Press, 2000: 287-310.

35. Jan D, Varad arajan R, Goldstein $M$ et al. Utilization of extended donor criteria liver allografts maximizes donor use and patient access to transplantation. Ann Surg 2005; 123: 250-259.

36. Matas AJ. The cases for living kidney sales: Rationale, objections, and concerns. Am J Transplant 2004; 4: 2007-2017.

37. Jha $\mathrm{V}$, Chugh KS. The case against a regulated system of living kidney sales. Nat Clin Pract Nephrol 2006; 2: 466-467.

38. Rothman SM, Rothman DJ. The hidden cost of organ sales. Am J Transplant 2006; 6: 1542-1528. 